\title{
Three-Dimensional Effects in High-Drag-State Flows over Long Ridges
}

\author{
Craig C. Epifanio and Dale R. Durran \\ Department of Atmospheric Sciences, University of Washington, Seattle, Washington
}

(Manuscript received 4 February 2000, in final form 22 August 2000)

\begin{abstract}
Numerical simulations of nonrotating flow with uniform basic wind and stability past long three-dimensional (3D) ridges are compared to the corresponding two-dimensional (2D) limit to reveal the importance of 3D effects. For mountain heights smaller than the threshold for breaking waves, the low-level flow over the interior of the ridge is well described by $2 \mathrm{D}$ theory when the horizontal aspect ratio $\beta$ is roughly 10 or greater. By contrast, in flows with wave breaking significant discrepancies between $2 \mathrm{D}$ and $3 \mathrm{D}$ results remain apparent even for $\beta$ $=12$.

It is found that the onset of wave breaking and the transition to the high-drag state is accompanied in 3D by an abrupt increase in deflection of the low-level flow around the ridge. The increased flow deflection is produced at least in part by upstream-propagating columnar disturbances forced by the transition to the high-drag state. The deflection of the incident flow reduces the amplitude of the mountain wave aloft relative to 2D and acts as a negative feedback on the surface form drag. As a result, the nonlinear enhancement of the surface drag associated with wave breaking for a ridge with $\beta=7.5$ is found to be roughly half the enhancement obtained for a $2 \mathrm{D}$ ridge.
\end{abstract}

\section{Introduction}

An extensive body of literature exists that explores both linear and nonlinear gravity waves generated by stratified flow over two-dimensional (2D) obstacles (see reviews in Smith 1979; Durran 1986b; Baines 1995). Among the interesting results to emerge from this work is the identification of highly nonlinear effects leading to flows with severe lee-slope winds and high surface form drag. Such high-drag states have been noted in flows with uniform basic wind and stability (Clark and Peltier 1977; Peltier and Clark 1979), with uniform wind and two layers of different stability (Durran 1986a), and with uniform stability and a basic-state critical level (Durran and Klemp 1987; Bacmeister and Pierrehumbert 1988). In each case, it is found that as a function of increasing mountain height, the flow first evolves through linear and weakly nonlinear wave regimes before making a relatively rapid transition to the fully nonlinear high-drag state. The finite-amplitude analytical model of Smith (1985) and the numerical investigations of Durran (1986a) and Durran and Klemp (1987) have suggested that the high-drag state is similar in character to nonlinear hydraulic flow with a subcritical to supercritical transition at the crest of the obstacle. As

Corresponding author address: Craig C. Epifanio, NCAR/MMMFL3, 3450 Mitchell Lane, Boulder, CO 80301.

E-mail: cepi@mmm.mmm.ucar.edu in the hydraulic case, the high-drag state is accompanied by upstream-propagating modes that decelerate the incident flow at low levels (Pierrehumbert and Wyman 1985; Baines 1995, sections 5.10, 5.11).

The linear theory of flow with uniform wind and stability over isolated three-dimensional (3D) obstacles was first examined in detail by Smith (1980, 1988, 1989b,a). Smith (1989a) identified four flow regimes based on the presence or absence of stagnation points in the flow: a weakly nonlinear regime in which flow stagnation does not occur, a wave-breaking regime with stagnation and overturned isentropes aloft, a flow-splitting regime featuring stagnation on the upwind slope, and a regime with both wave breaking and flow splitting. In this categorization of flow regimes, the presence of wave breaking is assumed to signal a high-drag state. ${ }^{1}$ By contrast, in the flow-splitting regime the majority of the low-level flow is assumed to deflect laterally around the barrier with limited vertical displacement, resulting in diminished wave amplitude aloft and reduced leeslope wind and surface form drag.

On the basis of the linear analysis, Smith (1989a) was able to construct an approximate regime diagram for

\footnotetext{
${ }^{1}$ In flows with uniform basic wind and stability, the transition to the high-drag state with increasing mountain height is coincident with the overturning of isentropes above the lee slope (Peltier and Clark 1979). As the present work is limited to uniform basic states, we use the terms high-drag state and wave-breaking regime interchangeably.
} 
elliptical mountains as a function of the nondimensional mountain height $\epsilon=N h_{0} / U_{0}\left(N\right.$ : basic stability, $U_{0}$ : basic wind speed, $h_{0}$ : maximum mountain height) and the horizontal aspect ratio $\beta$ (ratio of cross stream to streamwise length scales). For obstacles of fixed $\beta$ greater than unity, the regime diagram predicts that as a function of increasing $\epsilon$, the flow progresses successively through weakly nonlinear, wave-breaking, wavebreaking and flow-splitting, and flow-splitting-only regimes. For narrow mountains $(\beta<1)$, wave breaking is not predicted, and the flow progesses directly from the weakly nonlinear regime to the flow splitting regime.

Advances in computing power have facilitated a number of recent numerical studies of nonlinear flow with uniform wind and stability over 3D obstacles (e.g., Smolarkiewicz and Rotunno 1989, 1990; Miranda and James 1992; Smith and Grønås 1993; Ólafsson and Bougeault 1996, 1997; Schär and Durran 1997), which have largely confirmed the qualitative predictions of Smith's regime diagram. The majority of these studies have focused on inherently 3D effects in flow over circular mountains $(\beta=1)$. Smolarkiewicz and Rotunno (1990) considered elliptical mountains of varying $\beta$ but limited the investigation to large obstacles $(\epsilon=3)$ for which flow splitting is the dominant feature. Ólafsson and Bougeault (1996) examined each of the flow regimes for an elongated elliptical ridge but considered only a single fixed aspect ratio $(\beta=5)$. A recent study by Bauer et al. (2000) explores flow regimes for varying $\epsilon$ and $\beta$ but is limited to moderately elongated ridges $(\beta \leq 4)$.

Despite the recent effort devoted to exploring 3D flow over topography, there has been no systematic attempt to relate flow over obstacles in $3 \mathrm{D}$ to the well-studied 2D limit. Such a comparison is the objective of this study. We consider 3D ridges with relatively steep lateral ends connected by a uniform height section of variable length in the ridge interior. For large $\beta$ [e.g., $\beta \sim$ $O(10)]$, this $3 \mathrm{D}$ obstacle shape is expected to provide a more meaningful comparison to the $2 \mathrm{D}$ case over the ridge interior than the elliptical obstacles used in previous studies. The motivation for this work is twofold. First, we wish to determine the extent to which the 2D idealization provides a suitable approximation to flow over long (large $\beta$ ) ridges in 3D. Second, direct comparison of flow over long ridges to the 2D case highlights $3 \mathrm{D}$ effects, which are increasingly evident as $\epsilon$ increases.

The focus of the present study is on nonrotating weakly nonlinear and wave-breaking flows in a basic state of uniform wind and stability. Linear and weakly nonlinear steady-state semianalytic models as well as fully nonlinear numerical simulations are employed in an effort to highlight the nonlinear processes that shape the $3 \mathrm{D}$ flow. We note for clarity that the 3D effects of interest in this work are distinct from the 3D instability of breaking waves forced by the ridge, a topic that has received recent attention (Andreassen et al. 1994; Afanasyev and Peltier 1998). In the present numerical sim- ulations, the parameterized subgridscale turbulent fluxes of heat and momentum are sufficiently strong to prevent the resolved-scale waves from developing the streamwise vortices that are characteristic of 3D wave breakdown. We focus instead on more global properties of the flow, such as deflection of the low-level flow around the obstacle and the effect of such deflection on the wave amplitude and the surface drag.

In the following section we scale the equations of motion and briefly describe the semianalytic and numerical models. Section 3 examines the steady-state surface form drag and low-level flow deflection for 3D ridges of varying $\epsilon$ and $\beta$ and relates the results to the $2 \mathrm{D}$ case. It is found that for long $3 \mathrm{D}$ ridges wave breaking introduces significant 3D effects that are absent in weakly nonlinear flows. Some basic structural differences between high-drag-state flow over a long 3D ridge and the corresponding 2D case are discussed. Section 4 considers the role of upstream-propagating disturbances in establishing the 3D effects induced by wave breaking. Section 5 discusses possible extensions of the present work to flows with rotation and nonuniform basic states. The final section summarizes the results of the previous sections.

\section{Model descriptions}

a. Scale analysis and weakly nonlinear solution

We consider free-slip compressible-Boussinesq flow on an $f$ plane as described by

$$
\begin{gathered}
\frac{\partial u_{i}}{\partial t}+\left(u_{0}+u\right) \frac{\partial u_{i}}{\partial x}+\left(v_{0}+v\right) \frac{\partial u_{i}}{\partial y}+w \frac{\partial u_{i}}{\partial z} \\
+\epsilon_{i j k} f \delta_{j 3} u_{k}+\frac{\partial P}{\partial x_{i}}-\delta_{i 3} b=-\frac{\partial T_{i j}}{\partial x_{j}}, \\
\frac{\partial b}{\partial t}+\left(u_{0}+u\right) \frac{\partial b}{\partial x}+\left(v_{0}+v\right) \frac{\partial b}{\partial y}+w \frac{\partial b}{\partial z}+N^{2} w \\
=-\frac{\partial B_{j}}{\partial x_{j}}, \quad \frac{\partial P}{\partial t}+c_{s}^{2} \frac{\partial u_{j}}{\partial x_{j}}=0,
\end{gathered}
$$

with lower boundary condition

$$
w=\left(u_{0}+u\right) \frac{\partial h}{\partial x}+\left(v_{0}+v\right) \frac{\partial h}{\partial y} \quad \text { at } z=h .
$$

Here, $\mathbf{x}=\left(x_{1}, x_{2}, x_{3}\right)=(x, y, z)$ is the spatial position vector; $\mathbf{u}_{0}=\left(u_{0}, v_{0}, 0\right)$ is the uniform basic velocity; $\mathbf{u}=\left(u_{1}, u_{2}, u_{3}\right)=(u, v, w)$ is the disturbance velocity; $P$ is the Boussinesq disturbance pressure and $b$ the buoyancy; $T_{i j}$ and $B_{j}$ are turbulent fluxes of momentum and buoyancy, respectively; $N$ is the constant basic-state buoyancy frequency; $f$ is the Coriolis parameter; and $c_{s}$ is the constant Boussinesq sound speed. The topog- 
raphy is given by $h(x, y)$. Free-slip and thermal insulation conditions are adopted at the lower boundary for $T_{i j}$ and $B_{j}$, respectively. In a Boussinesq atmosphere, the amplitude of a localized 3D disturbance decays with height due to the horizontal dispersion of wave energy. The appropriate upper boundary is thus a linear radiation condition applied as $z \rightarrow \infty$.

Following Smith and Grønås (1993), we adopt an essentially linear, hydrostatic, nonrotating, inviscid scaling and define nondimensional independent and dependent variables by

$$
\begin{aligned}
& (x, y)=a(\hat{x}, \hat{y}), \\
& z=\frac{U_{0}}{N} \hat{z}, \quad t=\frac{a}{U_{0}} \hat{t}, \\
& \left(u_{0}, v_{0}\right)=U_{0}\left(\hat{u}_{0}, \hat{v}_{0}\right), \quad h=h_{0} \hat{h}, \quad w=\epsilon \delta U_{0} \hat{w}, \\
& (u, v)=\epsilon U_{0}(\hat{u}, \hat{v}), \quad P=\epsilon U_{0}^{2} \hat{P}, \quad b=\epsilon N U_{0} \hat{b},
\end{aligned}
$$

where $a$ is a characteristic length scale for $h, U_{0}=$ $\left(u_{0}^{2}+v_{0}^{2}\right)^{1 / 2}$ is the basic wind speed, $\delta=U_{0} / N a$ is the vertical aspect ratio, and $h_{0}$ and $\epsilon$ are as defined previously. Substituting the scaled variables into (1)-(4) yields the following set of control parameters: the nondimensional mountain height (or nonlinearity parameter) $\epsilon$, the vertical aspect ratio $\delta$, the Rossby number Ro $=U_{0} / f a$, the Mach number Ma $=U_{0} / c_{s}$, the angle of incidence $\psi=\arctan \left(\hat{v}_{0} / \hat{u}_{0}\right)$, and any parameters introduced in the specification of $\hat{h}$. A parameter describing the importance of the turbulent fluxes is also required to completely fix the flow. However, such a parameter is difficult to define generally, since the turbulent viscosity is a function of flow parameters such as the Richardson number and is also a function of grid resolution in the numerical context.

The linear and weakly nonlinear semianalytic models represent the first and second terms in a regular perturbation solution of the inviscid, incompressible (Ma $=0$ ), steady-state versions of (1)-(4) in the limit of small $\epsilon$. The solutions are formally valid for any finite $\delta$ and Ro. The models are implemented using fast Fourier transforms on a large horizontally periodic domain [as done for the linear solution by Smith (1980)]. Details of the weakly nonlinear model, including verifications, are given in Epifanio (1999) and will be summarized in a forthcoming publication.

\section{b. Numerical model and experimental set-up}

The fully nonlinear numerical simulations were computed using a 3D nested version of the nonhydrostatic model described by Durran and Klemp (1983) as modified to solve (1)-(3). Two-way interactive grid nesting is included following Skamarock and Klemp (1993). The model incorporates topography through the terrainfollowing vertical coordinate of Gal-Chen and Somerville (1975). The turbulent flux terms in (1) and (2) are computed using the first-order closure of Lilly (1962) with eddy length scale $(\Delta x \Delta z)^{1 / 2}$, mixing coefficient 0.21 , and Prandtl number unity. The linear radiation condition of Klemp and Durran (1983) and Bougeault (1983) (hereinafter the KDB condition) as modified for local evaluation by Durran (1995) is enforced at the upper boundary. A radiation condition with fixed-speed phase propagation is applied to normal velocities at the horizontal boundaries of the coarsest grid (Pearson 1974). In addition to the basic equations, we integrate

$$
\frac{\partial \zeta_{i}}{\partial t}+\left(u_{0}+u\right) \frac{\partial \zeta_{i}}{\partial x}+\left(v_{0}+v\right) \frac{\partial \zeta_{i}}{\partial y}+w \frac{\partial \zeta_{i}}{\partial z}=u_{i}
$$

where $\zeta=(\xi, \eta, \zeta)$ is the displacement of fluid particles from their undisturbed position following the basic flow.

The topography for all experiments takes the form of a smooth ridge with the long axis oriented perpendicular to the $x$ direction as defined by

$$
h(x, y)= \begin{cases}\frac{h_{0}}{16}[1+\cos (\pi r)]^{4}, & \text { if } r \leq 1 \\ 0, & \text { otherwise }\end{cases}
$$

where

$$
r^{2}= \begin{cases}\left(\frac{x}{4 a}\right)^{2}+\left(\frac{|y|-(\beta-1) a}{4 a}\right)^{2}, & \text { if }|y|>(\beta-1) a \\ \left(\frac{x}{4 a}\right)^{2}, & \text { otherwise. }\end{cases}
$$

Here, $\beta \geq 1$ is the horizontal aspect ratio defined as the ratio of $y$ to $x$ length scales for the topography. For $\beta$ $=1, h(x, y)$ is similar to a bell-shaped mountain [as in Smith (1980)] with length scale $a$; for $\beta>1$, a uniform height section appears in the interior of the ridge.

We consider essentially hydrostatic $(\delta=0.067)$, incompressible $(\mathrm{Ma}=0.024)$, nonrotating $(f=0)$ flow at normal incidence $\left(v_{0}=0\right)$. The set of control parameters then reduces to $\epsilon$ and $\beta$ (for fixed turbulence parameterization), and the values of these parameters considered in this study are shown in Table 1 . The specific dimensional parameters used are $N=0.012 \mathrm{~s}^{-1}, U_{0}=$ $8 \mathrm{~m} \mathrm{~s}^{-1}$, and $a=10 \mathrm{~km}$. The disturbance is initiated by gradually accelerating the basic wind from rest over the time interval $-4 \leq U_{0} t / a \leq 0$.

Three levels of nesting are employed with horizontal and temporal resolution increasing by a factor of 3 with each nesting level. The grid with finest horizontal mesh occupies the region $|x| \leq 7 a,|y| \leq(6+\beta) a$; the intermediate grid covers $|x| \leq 18 a,|y| \leq(17+\beta) a$; and the coarse grid extends over $|x| \leq 48.6 a,|y| \leq 48.6 a$. The horizontal mesh spacing on the finest grid is $\Delta x=$ $\Delta y=0.18 a$. Vertical resolution and domain depth $z_{T}$ are identical on all grids and are given by $N \Delta z / U_{0}=$ $0.086 \pi$ and $N z_{T} / U_{0}=8.4 \pi$. The large and small time 
TABLE 1. Values of $\epsilon$ and $\beta$ for numerical simulations in this study ( $\times$ indicates simulated value).

\begin{tabular}{|c|c|c|c|c|c|c|c|}
\hline \multirow[b]{2}{*}{$\epsilon$} & \multicolumn{6}{|c|}{$\beta$} & \multirow[b]{2}{*}{$2 \mathrm{D}$} \\
\hline & 1.00 & 1.54 & 2.38 & 3.75 & 7.50 & 12.0 & \\
\hline 1.5 & & & & & $x$ & & $x$ \\
\hline 1.4 & & & & & $x$ & & $\times$ \\
\hline 1.3 & & & & & $\times$ & & $x$ \\
\hline 1.2 & & & & & $\times$ & & $x$ \\
\hline 1.1 & & & & & $\times$ & & $\times$ \\
\hline 1.0 & $x$ & $\times$ & $\times$ & $x$ & $\times$ & $\times$ & $x$ \\
\hline 0.9 & & & & & $\times$ & & $\times$ \\
\hline 0.8 & & & & & $x$ & & $x$ \\
\hline 0.7 & & & & & $\times$ & & $\times$ \\
\hline 0.6 & & & & & $\times$ & & $\times$ \\
\hline 0.5 & $\times$ & $\times$ & $\times$ & $\times$ & $\times$ & $\times$ & $x$ \\
\hline 0.4 & & & & & $x$ & & $x$ \\
\hline 0.3 & & & & & $x$ & & $x$ \\
\hline 0.2 & & & & & $\times$ & & $x$ \\
\hline 0.1 & & & & & $\times$ & & $\times$ \\
\hline
\end{tabular}

steps on the finest mesh are $U_{0} \Delta t / a=0.0056$ and $U_{0} \Delta t_{s} / a=0.0056 / 3$, respectively. ${ }^{2}$

\section{c. Model sensitivities}

The influence of horizontal periodicity on the weakly nonlinear semianalytic model was minimized by repeating the computation on square domains of increasing size until the nonlinear correction to the drag $\left[\hat{C}_{D}^{(2)}\right.$ in (11)] varied by less than $1 \%$ between successive computations. The weakly nonlinear solution also entails a linear radiation condition, which in principle is applied for $z \rightarrow \infty$ but in practice must be applied at a large but finite height. The radiation condition at finite height is essentially equivalent to the KDB condition employed in the numerical simulations. For the present experiments, the radiation condition is applied at the same height $z_{T}$ for both the numerical and weakly nonlinear semianalytic models so as to remove the upper boundary as a source of discrepancy between the solution techniques. The sensitivity of the weakly nonlinear solution to the height at which the radiation condition is applied will be discussed in upcoming work.

As discussed in Klemp and Durran (1983), neither the KDB radiation condition nor alternative sponge-layer formulations completely eliminate the sensitivity of numerically simulated mountain waves to the location of the upper boundary when the waves are nonlinear (e.g., $\epsilon \gtrsim 0.5$ ). The upper boundary sensitivity of our numerical model for large $\epsilon$ is illustrated in Fig. 1. Figure 1a shows the time dependence of the surface form drag $\left[\hat{C}_{D}\right.$ in (9)] for flow over a 3D ridge with $\epsilon=1$ and $\beta=7.5$ for various domain depths $z_{T}$. The surface

\footnotetext{
${ }^{2}$ As described in Durran and Klemp (1983), the compressible numerical model maintains stability by integrating the terms responsible for acoustic propagation on a small time step $\Delta t_{s}$ and the remaining terms on a larger step $\Delta t$.
}
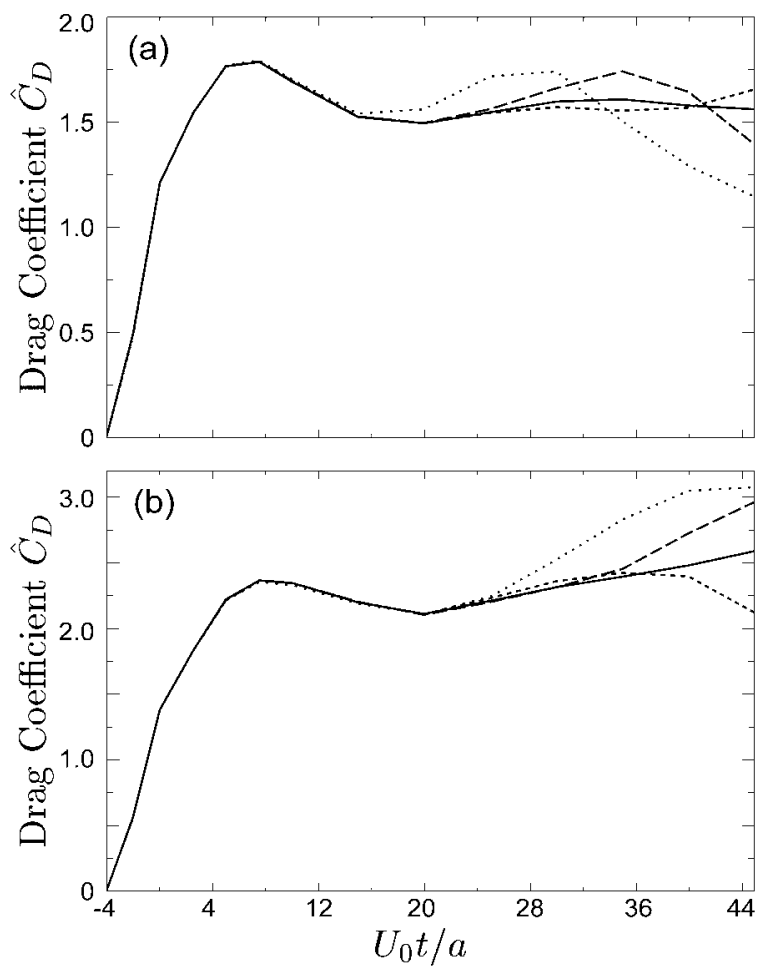

FIG. 1. (a) Time dependence of the nondimensional drag coefficient $\hat{C}_{D}$ for $\epsilon=1, \beta=7.5$, and domain depths $N z_{T} / U_{0}=4.3 \pi$ (dotted), $6.4 \pi$ (long dashed), $7.4 \pi$ (dashed), and $8.4 \pi$ (solid). (b) As in (a) except 2D case.

drags for all domain depths are identical until the influence of the upper boundary becomes significant, at which point the simulated drags diverge. In a 3D Boussinesq atmosphere, increasing the depth of the numerical domain improves the performance of the upper boundary in two ways: greater domain depth gives an increased delay time before reflections from the upper boundary become significant near the surface, and increasing the upper boundary height decreases the amplitude of the disturbance near the boundary (due to 3D wave dispersion) and thus improves the effectiveness of the radiation condition. Figure 1a suggests that a domain depth $N z_{T} / U_{0} \approx 4 \pi$ is insufficient to achieve a realistic approach to steady state for flow over long ridges. By contrast, the domain depth $N z_{T} / U_{0}=8.4 \pi$ used in the present study is seen to be quantitatively accurate to at least time $U_{0} t / a=35$ and qualitatively reasonable for the duration of the present simulations (which terminate at $U_{0} t / a=45$ ).

In contrast to the 3D case, 2D hydrostatic disturbances in a Boussinesq atmosphere do not decay in amplitude with height. As such, increasing the domain depth in the 2D case simply increases the delay time before reflections from the upper boundary corrupt the solution near the surface. Nonetheless, the time-dependent surface form drags shown in Fig. 1b suggest that even in the $2 \mathrm{D}$ case, the domain depth $N z_{T} / U_{0}=8.4 \pi$ 
provides a sufficiently accurate solution to at least time $U_{0} t / a=35$.

The coarse and nested grids were found to be of sufficient horizontal extent to minimize sensitivity to lateral grid boundaries.

\section{Quasi-steady surface drag and flow deflection}

This section explores the surface form drag and lowlevel flow deflection generated by ridges of varying $\epsilon$ and $\beta$ at times long after the initial transient growth of the disturbance. Note that even for the long time integrations considered here, the flow in most cases does not achieve a completely steady state. As such, there is necessarily a degree of subjectivity in choosing representative quasi-steady values for the surface drag and low-level mass deflection (defined in section $3 \mathrm{~b}$ ). In the present work, the surface drags reported as steady-state in section 3 a have been evaluated visually based on the behavior of the drag after the initial transient growth phase. Appendix A gives several examples showing the time variation of the drag after the initial growth phase and the chosen representative quasi-steady values. For most of the $3 \mathrm{D}$ ridge cases and for the $2 \mathrm{D}$ cases without breaking waves, the drag is relatively steady after $U_{0} t / a$ $=20$ and the representative quasi-steady value is determined with good precision (see appendix A for details). By contrast, in the 2D cases with wave breaking, the surface drag steadily amplifies after $U_{0} t / a=20$ and the flow does not reach a quasi-steady state. The drag at $U_{0} t / a=32.5$ is then taken as a representative value.

The low-level deflected mass fluxes considered in section $3 \mathrm{~b}$ become relatively steady after the initial transient phase in cases without breaking waves. In cases with wave breaking, the deflected mass fluxes increase monotonically with time but generally become quasisteady near the end of the simulation. In most cases, the deflected mass flux is taken as approximately steady at $U_{0} t / a=35$. Exceptions are the calculations for $\beta=$ 7.5 and $\epsilon=0.8$ and 0.9. For $\epsilon=0.9$, the mass flux at $U_{0} t / a=40$ is taken as steady. For $\epsilon=0.8$, the deflected mass flux increases for the duration of the simulation without reaching a quasi-steady state, and the value at the end of the simulation $\left(U_{0} t / a=45\right)$ is taken as representative.

The time dependence of the surface drag and lowlevel flow deflection are considered in more detail in section 4.

\section{a. Surface form drag}

The surface form drag

$$
D=\int_{-\infty}^{\infty} \int_{-\infty}^{\infty} P \frac{\partial h}{\partial x} d x d y
$$

depends both on the pressure difference across the barrier and on the cross-sectional area of the barrier in the plane normal to the incident flow. To remove the explicit dependence of the drag on the obstacle cross section and to allow direct comparison of flows over ridges of varying lengths, we define the dimensional drag coefficient as

$$
\begin{aligned}
& C_{D}= \frac{\int_{-\infty}^{\infty} \int_{-\infty}^{\infty} P(x, y, h) \frac{\partial h}{\partial x} d x d y}{\int_{-\infty}^{\infty} h(0, y) d y} \\
&=\epsilon U_{0}^{2} \frac{\int_{-\infty}^{\infty} \int_{-\infty}^{\infty} \hat{P}(\hat{x}, \hat{y}, \epsilon \hat{h}) \frac{\partial \hat{h}}{\partial \hat{x}} d \hat{x} d \hat{y}}{\int_{-\infty}^{\infty} \hat{h}(0, \hat{y}) d \hat{y}}=\epsilon U_{0}^{2} \hat{C}_{D},
\end{aligned}
$$

where the denominator is the cross-sectional area of the obstacle. The dimensional drag coefficient measures the average pressure difference across the barrier and thus has the same linear scaling as pressure.

In the context of the linear and weakly nonlinear solutions, we may expand $\hat{P}(\hat{x}, \hat{y}, \epsilon \hat{h})$ in powers of $\epsilon$ as

$$
\begin{aligned}
\hat{P}(\hat{x}, \hat{y}, \epsilon \hat{h})= & \hat{P}^{(1)}(\hat{x}, \hat{y}, 0) \\
& +\epsilon\left[\hat{P}^{(2)}(\hat{x}, \hat{y}, 0)+\hat{h} \frac{\partial \hat{P}^{(1)}}{\partial \hat{z}}(\hat{x}, \hat{y}, 0)\right]+\cdots,
\end{aligned}
$$

and substitute into (9) to find

$$
C_{D}=\epsilon U_{0}^{2} \hat{C}_{D}=\epsilon U_{0}^{2}\left(\hat{C}_{D}^{(1)}+\epsilon \hat{C}_{D}^{(2)}+\cdots\right) .
$$

The linear and weakly nonlinear semianalytic models predict the first and second terms of this expansion, respectively.

The nondimensional coefficient $\hat{C}_{D}$ is a stratified analog [for the scaling in (5)] of the standard drag coefficient defined for homogenous flows past bluff bodies (e.g., Batchelor 1967, his section 5.11), and in the following, we refer to $\hat{C}_{D}$ simply as the drag coefficient. Note that in (11), the coefficients $\hat{C}_{D}^{(1)}, \hat{C}_{D}^{(2)}, \ldots$ are independent of $\epsilon$ and thus depend only on $\beta$. In particular, for a given $\beta$, the linear prediction $\hat{C}_{D}^{(1)}$ is independent of $\epsilon$ so that any variation of $\hat{C}_{D}$ with $\epsilon$ is due entirely to nonlinear effects. According to (11), the first nonlinear correction to $\hat{C}_{D}$ as predicted by weakly nonlinear theory varies linearly with $\epsilon$ for fixed $\beta$.

Figure $2 \mathrm{a}$ shows the aspect ratio dependence of the drag coefficient as predicted by the linear, weakly nonlinear, and fully nonlinear models for $\epsilon=0.5$. The drag coefficient for a 2D ridge, defined from the $2 \mathrm{D}$ limit of (9) as

$$
\hat{C}_{D}=\int_{-\infty}^{\infty} \hat{P}(\hat{x}, \epsilon \hat{h}) \frac{\partial \hat{h}}{\partial \hat{x}} d \hat{x}, \quad \text { (2D case), }
$$

is indicated by a thin horizontal line for the linear and fully nonlinear solutions. (The 2D drag coefficient predicted by the weakly nonlinear model is not believed 

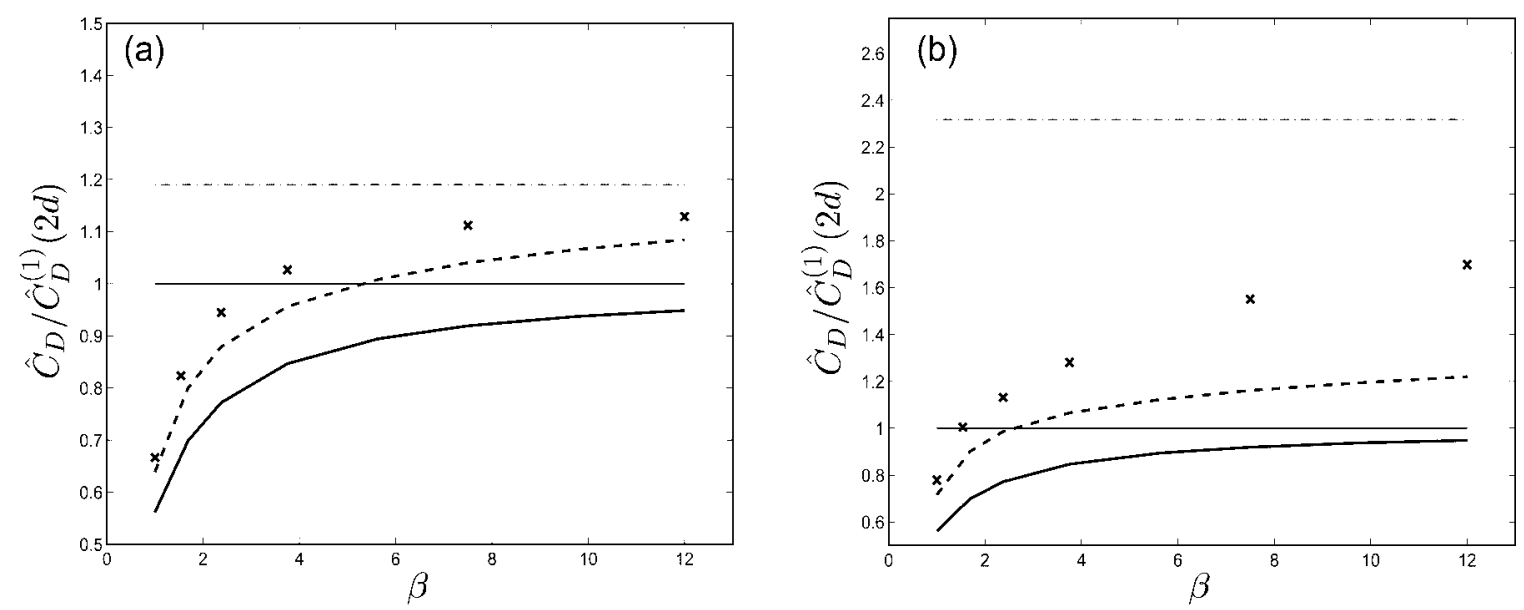

FIG. 2. (a) Normalized drag coefficient as a function of $\beta$ for $\epsilon=0.5$ as predicted by linear theory (solid), weakly nonlinear theory (dashed), and fully nonlinear numerical simulation (crosses). The normalized drag coefficient for the 2D case is shown as a thin horizontal line for the linear theory (solid) and nonlinear numerical (dot-dashed) cases. (b) As in (a) except $\epsilon=1.0$.

to be relevant due to the inapplicability of the linear radiation condition in the nonlinear 2D steady-state problem.) In all cases, the drag coefficient has been normalized using the linear coefficient for 2D flow, ${ }^{3}$ $\hat{C}_{D}^{(1)}(2 d)=1.02$. As expected, the drag coefficient for an axisymmetric $(\beta=1)$ obstacle is significantly weaker than the $2 \mathrm{D} \hat{C}_{D}$ for each of the linear, weakly nonlinear, and nonlinear solutions. However, the drag coefficient for the linear and nonlinear models does appear to asymptote to the $2 \mathrm{D}$ result as $\beta$ increases. For $\beta=12$, the difference between the $2 \mathrm{D}$ and 3D drag coefficients ${ }^{3}$ We do not have analytic expressions for the drag generated by
the obstacle ( 7 ). As such, the $2 \mathrm{D}$ value $\hat{C}_{D}^{(1)}=1.02$ is simply the
result predicted by the linear semianalytic model.

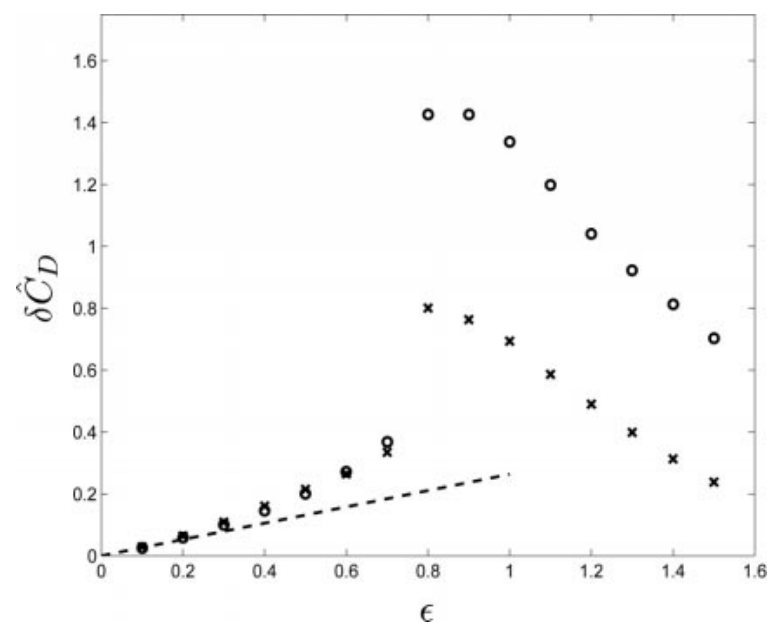

FIG. 3. Nonlinear drag amplification $\delta \hat{C}_{D}$ as a function of $\epsilon$ for the fully nonlinear $2 \mathrm{D}$ (circles) and $\beta=7.5$ (crosses) calculations and for the $\beta=7.5$ weakly nonlinear semianalytic model (dashed). is roughly $5 \%$ of the $2 \mathrm{D}$ value for both the linear and fully nonlinear solutions.

For $\epsilon=0.5$, the flow is in the weakly nonlinear regime for all aspect ratios, and the nonlinear drag is well represented by the second-order weakly nonlinear solution. Figure $2 \mathrm{~b}$ shows the corresponding situation for $\epsilon=1.0$. At this mountain height, isentropes above the lee slope overturn and wave breaking occurs for $\beta$ slightly greater than unity. With the onset of wave breaking, the flow becomes highly nonlinear, and the weakly and fully nonlinear models yield very different solutions. Significantly, in contrast to the $\epsilon=0.5$ case, the fully nonlinear $\hat{C}_{D}$ for $\epsilon=1.0$ remains well below the nonlinear $2 \mathrm{D}$ result for $\beta$ at least as large as 12 . Threedimensional effects are thus evident in the highly nonlinear wave-breaking regime even for relatively long ridges.

As suggested by Fig. 2b, the nonlinear amplification of the drag coefficient associated with the onset of wave breaking in flows with constant $N$ and $U_{0}$ is significantly weaker for flow over $3 \mathrm{D}$ ridges than for $2 \mathrm{D}$ flows. Figure 3 shows the nonlinear amplification factor

$$
\delta \hat{C}_{D}=\frac{\hat{C}_{D}-\hat{C}_{D}^{(1)}}{\hat{C}_{D}^{(1)}}
$$

as a function of $\epsilon$ for flow over a ridge with $\beta=7.5$ and for $2 \mathrm{D}$ flow. Here, $\hat{C}_{D}^{(1)}$ refers to the linear drag coefficient corresponding to the particular case (i.e., 2D or $\beta=7.5)$. The dashed line represents the nonlinear amplification predicted by the weakly nonlinear model for $\beta=7.5$, as given by substituting the first two terms of the expansion in (11) into (12). ${ }^{4}$ The increase in the

\footnotetext{
${ }^{4}$ Note the excellent agreement between the nonlinear numerical and weakly nonlinear semianalytic predictions for $\delta \hat{C}_{D}$ in the limit
} 

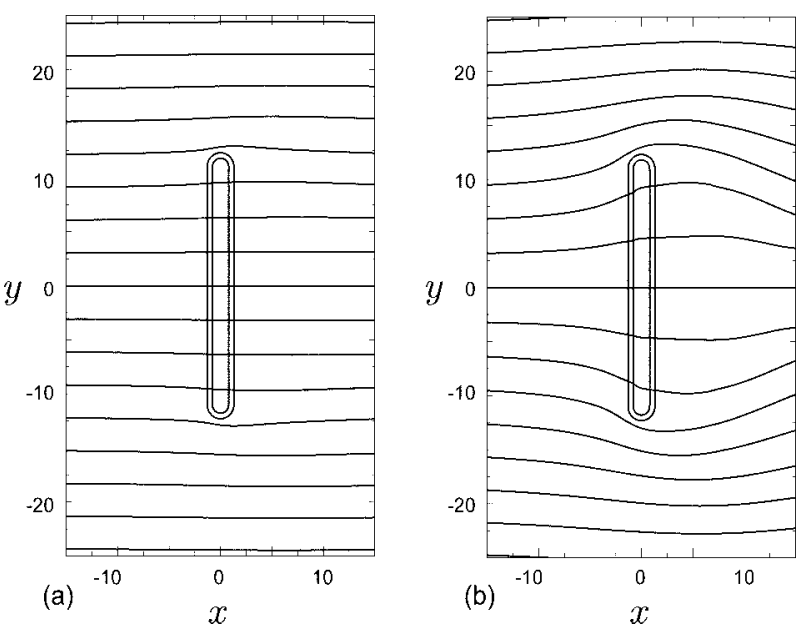

FIG. 4. Lateral deviation of initially streamwise dye lines on the low-level material surface $N(z-\zeta) / U_{0}=0.4$ for a ridge with $\beta=$ 12 and (a) $\epsilon=0.5$ or (b) $\epsilon=1.0$.

nonlinear amplification factor (12) associated with the onset of wave breaking at $\epsilon \approx 0.8$ is readily apparent in both the $2 \mathrm{D}$ and $\beta=7.5$ simulations. However, the increase in $\delta \hat{C}_{D}$ in the $3 \mathrm{D}$ case is much reduced from the corresponding $2 \mathrm{D}$ result. At mountain height $\epsilon=$ 0.8 , the nonlinear amplification $\delta \hat{C}_{D}$ for $\beta=7.5$ is roughly $55 \%$ of the $2 \mathrm{D}$ value.

\section{b. Low-level mass fluxes}

The reduction in the nonlinear enhancement of the drag in the wave-breaking regime in $3 \mathrm{D}$ appears to be related to an increase in the lateral deflection of the lowlevel flow associated with wave breaking in the finitelength ridge case. Figure 4 shows initially streamwise dye lines [i.e., lines of constant $y-\eta$; see (6)] on the low-level material surface $N(z-\zeta) / U_{0}=0.4$ at time $U_{0} t / a=35$ for the case $\beta=12$. As illustrated in the figure, the lateral deviation of the dye lines increases dramatically as $\epsilon$ increases from 0.5 to 1.0.

A quantitative measure of the low-level flow deflection can be obtained by considering a mass budget for the control volume shown schematically in Fig. 5. The lower boundary of the control volume coincides with the topography, the sides are embedded in vertical planes, and the top boundary is an initially horizontal material surface $z-\zeta=z_{0}$ where $z_{0}$ is a constant. The sides parallel to the $x$ axis intersect the ends of the uniform height section of the ridge at $y= \pm(\beta-1) a$. The downstream side is located at the obstacle crest $x$ $=0$, and the upstream face is placed as far upstream

of small $\epsilon$ for the $\beta=7.5$ case (Fig. 3). To obtain this agreement, it was necessary to compute the numerical solutions for $\epsilon=0.1,0.2$, and 0.3 using double the standard machine precision to capture the weak nonlinear effects at small $\epsilon$.

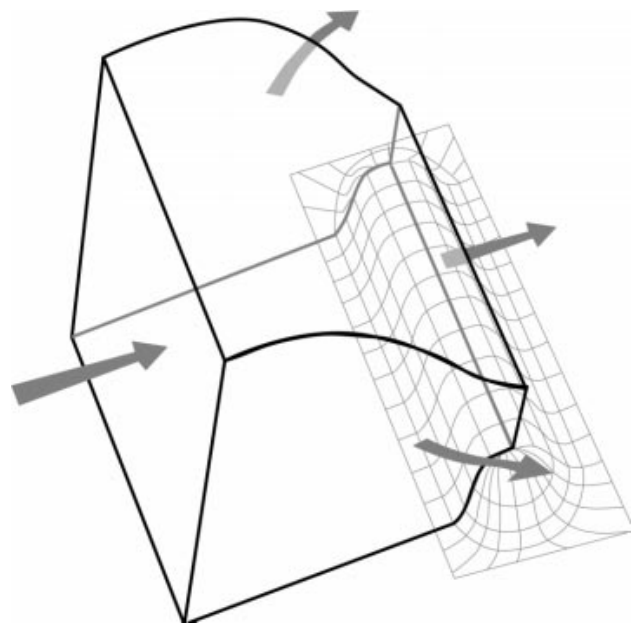

FIG. 5. Control volume for mass flux calculations. See text for details.

as is computationally feasible, which in this case was $x=-15 a$. Note that the upper and lower boundaries of the control volume are impermeable to mass fluxes. As such, the mass entering the control volume through the upstream face at steady state must either flow over the obstacle crest or be deflected laterally out of the control volume and around the ridge.

Let $\tilde{z}\left(x, y, z_{0}\right)$ denote the height of the material surface with initial undisturbed height $z_{0}$ [i.e., $\tilde{z}\left(x, y, z_{0}\right)=z_{0}$ $+\zeta(x, y, z)]$, and consider the mass fluxes

$$
\begin{aligned}
& F_{y}\left(y, z_{0}\right)=\int_{-15 a}^{0} \int_{h}^{z\left(z_{0}\right)} \rho_{s} v d z d x, \\
& F_{x}\left(x, z_{0}\right)=\int_{-(\beta-1) a}^{(\beta-1) a} \int_{h}^{z\left(z_{0}\right)} \rho_{s}\left(u+u_{0}\right) d z d y,
\end{aligned}
$$

where $\rho_{s}$ is a constant reference density for the Boussinesq system. A measure of the flow deflection due to the ridge may then be defined as

$$
\begin{aligned}
D_{M} & =\frac{F_{y}\left[(\beta-1) a, z_{0}\right]-F_{y}\left[-(\beta-1) a, z_{0}\right]}{F_{x}\left(-15 a, z_{0}\right)} \\
& =\epsilon \hat{D}_{M} .
\end{aligned}
$$

The fractional mass deflection $D_{M}$ can be interpreted as the fraction of the upstream mass flux below $z_{0}$ incident on the uniform height section of the ridge that deflects around the ridge rather than ascending the crest. Nonlinear effects are highlighted by normalizing $D_{M}$ by its linear scaling to remove the $\epsilon$ dependence predicted by linear theory. The scaled fractional mass deflection $\hat{D}_{M}$ may then be expanded in terms of $\epsilon$, as in (11), to achieve linear and weakly nonlinear approximations. However, the weakly nonlinear approximation to $\hat{D}_{M}$ is rather arduous to compute, and only the linear mass 


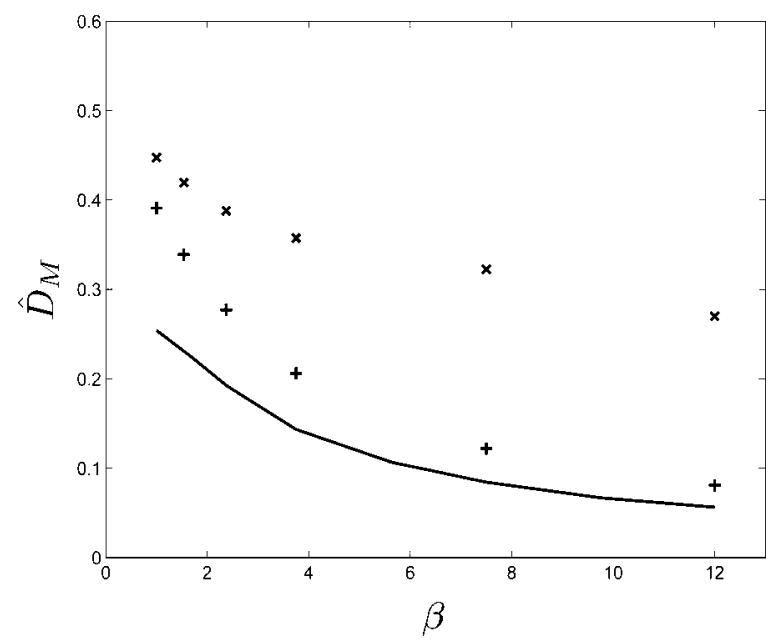

FIG. 6. Scaled low-level fractional mass deflection $\hat{D}_{M}$ as a function of $\beta$ for $\epsilon=0.5$ (plus signs) and $\epsilon=1.0$ (crosses). Solid line shows linear prediction $\hat{D}_{M}^{(1)}$.

deflection $\hat{D}_{M}^{(1)}$ is calculated here. ${ }^{5}$ For the case $\beta=1$, the mountain is circular, and we consider the limiting form of (14)

$$
D_{M}=\frac{\int_{-15 a}^{0} \int_{h}^{z\left(z_{0}\right)} \rho_{s} \frac{\partial v}{\partial y} d z d x}{\int_{h}^{z\left(z_{0}\right)} \rho_{s}\left(u+u_{0}\right) d z} \quad(\beta=1 \text { case }),
$$

where all quantities are evaluated at $y=0$. In the following, we have chosen the bounding surface $N z_{0} / U_{0}$ $=2$, in which case the top of the control volume is coincident with the lowest dye line plotted in Figs. 9 and 10. Above the lee slope, this surface is roughly aligned with the top of the region of highly accelerated downslope flow in cases with wave breaking.

The influence of aspect ratio on low-level flow deflection is illustrated in Fig. 6, which shows the scaled fractional mass deflection $\hat{D}_{M}$ as a function of $\beta$ for $\epsilon$ $=0.5$ and $\epsilon=1.0$. The linear prediction $\hat{D}_{M}^{(1)}$ is shown as a solid line. As expected, the fractional mass deflection decreases sharply with increasing $\beta$ for the linear and $\epsilon=0.5$ calculations. At $\beta=12$ and $\epsilon=0.5$, less than $5 \%$ of the incident mass flux below $z_{0}$ is deflected around the ridge (i.e., $D_{M}=\epsilon \hat{D}_{M}<0.05$ ). The fractional mass deflection decreases much less rapidly with $\beta$ for the $\epsilon=1.0$ case. Even at $\beta=12$, nearly $30 \%$ of the incident mass flux below $z_{0}$ is deflected laterally around the ridge for $\epsilon=1.0$.

\footnotetext{
${ }^{5}$ The linear fractional mass deflection $\hat{D}_{M}^{(1)}$ is computed by first calculating the linear semianalytic solution on a regular grid and then computing the linear approximations to $F_{\mathrm{y}}\left[ \pm(\beta-1) a, z_{0}\right]$ numerically from the gridded data. [In the linear approximation to (14), the denominator is taken to be $2 \rho_{s} u_{0} z_{0}(\beta-1) a$.] The linear approximation to (15) is computed similarly.
}

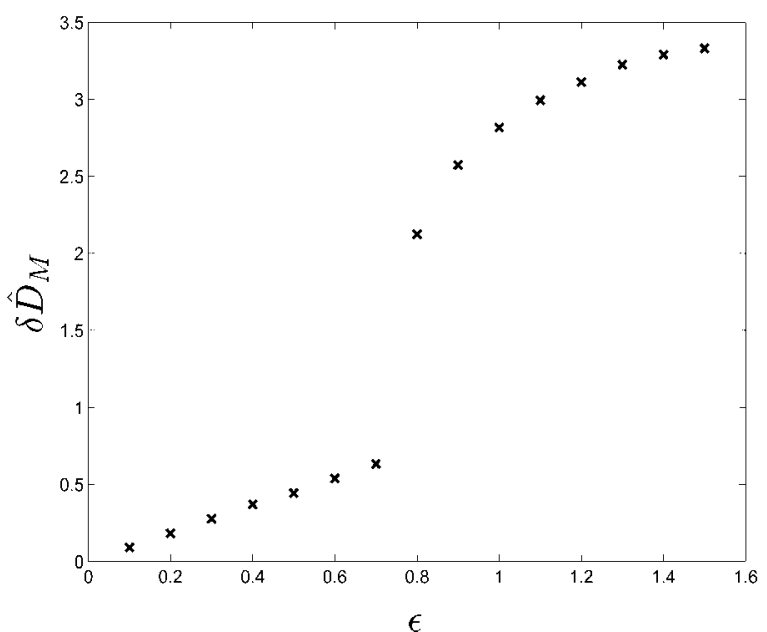

FIG. 7. Nonlinear enhancement of the low-level mass deflection $\delta \hat{D}_{M}$ as a function of $\epsilon$ for the case $\beta=7.5$.

The results of Fig. 6 suggest that the increased nonlinearity associated with wave breaking has a profound effect on the lateral deflection of the low-level flow. The influence of wave breaking on the low-level flow deflection is seen more clearly in Fig. 7, which shows the nonlinear enhancement of the mass deflection

$$
\delta \hat{D}_{M}=\frac{\hat{D}_{M}-\hat{D}_{M}^{(1)}}{\hat{D}_{M}^{(1)}},
$$

[cf. (12)] as a function of $\epsilon$ for $\beta=7.5$. A comparison of Fig. 7 with Fig. 3 indicates that the onset of wave breaking at $\epsilon \approx 0.8$ leads to a significant increase in the nonlinear contribution to the low-level mass deflection as well as the surface form drag. It is likely that the increased flow deflection associated with wave breaking acts as a negative feedback on the surface drag and at least partially accounts for the discrepancy between the 2D and 3D results in Figs. 2b and 3. The relationship between surface drag and low-level flow deflection is explored in more detail in the following subsection.

\section{c. Wave amplitude and lee-slope warming}

The pronounced lateral deflection of the low-level flow upstream of the obstacle in 3D cases with breaking waves leads to stronger downward displacements in the lee as air from aloft descends to replace the deflected mass. As a consequence, the near-surface flow above the lee slope along the interior of a $3 \mathrm{D}$ ridge is more positively buoyant than the corresponding 2D flow. Figure 8 compares the buoyancy at the lowest thermodynamic grid level $\left(N z / U_{0} \approx \pi / 23\right)$ in the $\epsilon=1.02 \mathrm{D}$ case with that averaged over the uniform height section [i.e., over $-(\beta-1) a \leq y \leq(\beta-1) a]$ of a 3D ridge with $\epsilon=1.0$ and $\beta=7.5$. The lee-side buoyancy is significantly greater in the 3D case than in the $2 \mathrm{D}$ case, and the increase in buoyancy is maintained well downstream 


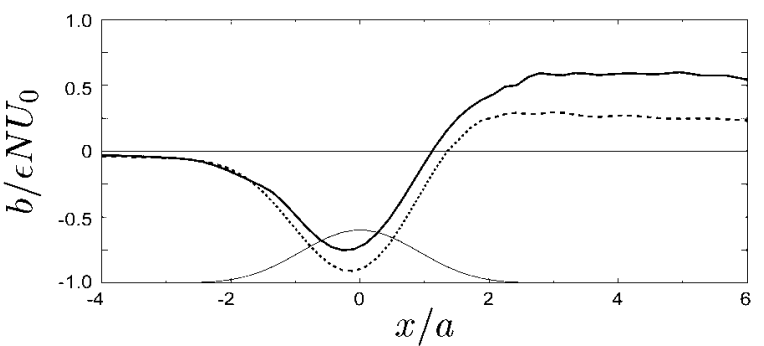

FIG. 8. Buoyancy $b / \epsilon N U_{0}$ at the lowest thermodynamic grid level $\left(N z / U_{0} \approx \pi / 23\right)$ at time $U_{0} t / a=35$. Solid line shows average over the uniform height section [i.e., over $-(\beta-1) a \leq y \leq(\beta-1) a$ ] of a 3D ridge with $\beta=7.5$ and $\epsilon=1.0$ and dashed line is the corresponding $2 \mathrm{D}$ case.

of the obstacle. The enhancement of the buoyancy over the lee slope in the 3D flow is similar to the föehn effect often observed in flows over mountain ranges.

Figure 9 shows the scaled buoyancy $b / \epsilon N U_{0}$ in vertical cross section for $\epsilon=1.0$ at time $U_{0} t / a=35$ for the $2 \mathrm{D}$ case and along the ridge centerline for the cases $\beta=12$ and $\beta=7.5$. A comparison of Figs. 9a, 9b, and $9 \mathrm{c}$ indicates that although the near-surface lee-slope buoyancy is increased in the 3D simulations relative to the $2 \mathrm{D}$ case, the buoyancy further aloft above the lee slope is significantly greater for the $2 \mathrm{D}$ case throughout most of the lowest wavelength of the disturbance (approximately throughout the region $0.125 \leq N z / 2 \pi U_{0} \leq$ $0.75)$. Similarly, comparison of the dye lines in Figs. $9 \mathrm{a}, 9 \mathrm{~b}$, and $9 \mathrm{c}$ suggests that the deflection of the lowlevel flow upstream of the 3D ridges results in reduced vertical displacements above the windward slope and weaker wave amplitude aloft. The weaker wave amplitude and accompanying reduction in vertically integrated buoyancy above the lee slope results in a weaker lee-slope pressure minimum in the $\beta=12$ and $\beta=7.5$ cases relative to $2 \mathrm{D}$, as shown in Fig. 10 . The pronounced weakening of the lee-side pressure minimum primarily accounts for the reduction in the surface form drag in 3D simulations with wave breaking relative to the $2 \mathrm{D}$ case as discussed in section $3 \mathrm{a}$.

\section{Upstream influence and low-level flow deflection}

The onset of wave breaking and the transition to the high drag state in stratified flow produces upstreampropagating disturbances in a manner analogous to the generation of upstream disturbances by hydraulic transitions in flows of multiple homogenous layers. Such disturbances are dominated by nearly columnar modes (i.e., modes with $x$-direction wavenumber $k \approx 0$ and vertical group speed $c_{g z} \approx 0$ ), which act to decelerate and deepen the low-level flow upstream. Upstream disturbances in 2D stratified flow have received detailed attention in previous studies (for review, see Baines 1995, sections 5.11 and 5.12). Here, we briefly compare the upstream disturbances generated by $2 \mathrm{D}$ and $3 \mathrm{D}$ ridg-
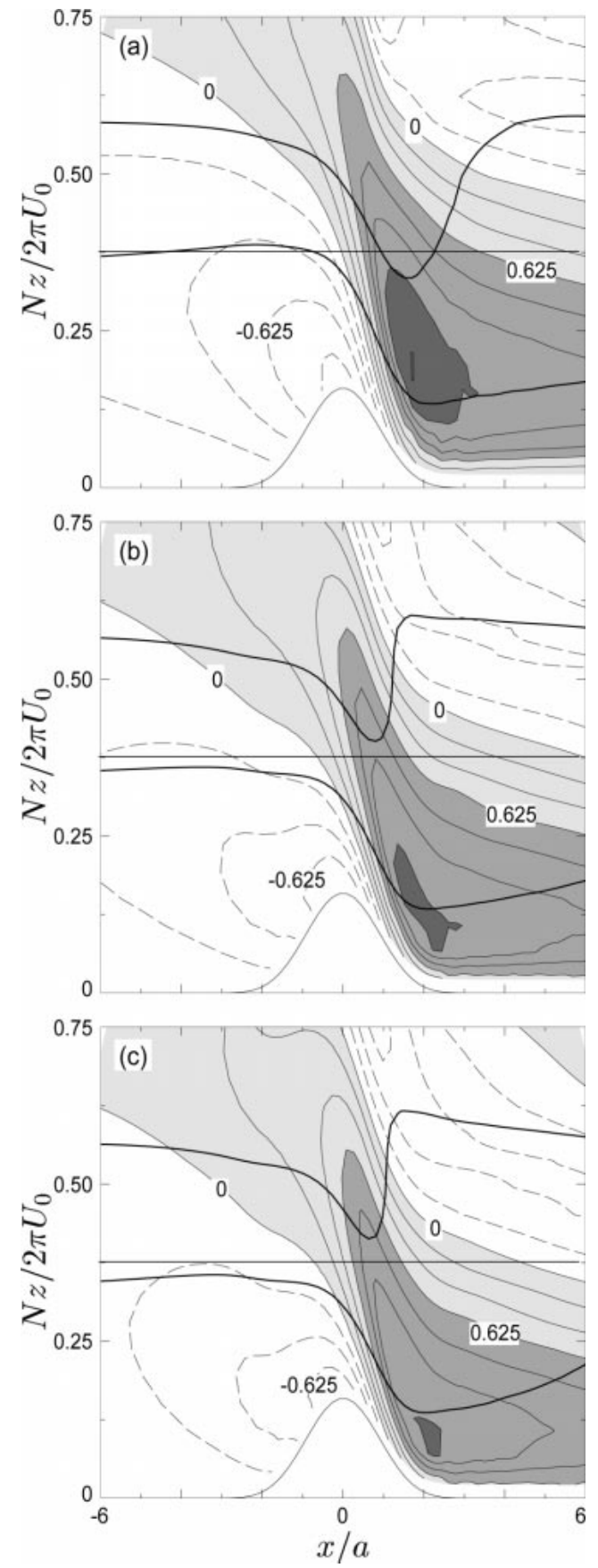

FIG. 9. Buoyancy $b / \epsilon N U_{0}$ contoured at intervals of 0.2083 at $U_{0} t / a$ $=35$ for (a) flow over a $2 \mathrm{D}$ ridge and for flow along the centerline of 3D ridges with (b) $\beta=12$ and (c) $\beta=7.5$. In all cases, $\epsilon=1.0$. Darkest shading indicates values in excess of 1.25. Overlaid as thick solid lines are dye lines corresponding to material surfaces with $N(z$ $-\zeta) / U_{0}=2$ and $N(z-\zeta) / U_{0}=3.5$. Thin horizontal lines at $N z / U_{0}$ $=0.75 \pi$ are shown for reference.

es and consider the effect of such disturbances on the 3D flow.

Figure 11a shows the disturbance field $u / \epsilon U_{0}$ upstream of the obstacle crest at $U_{0} t / a=40$ for the $2 \mathrm{D}$ 

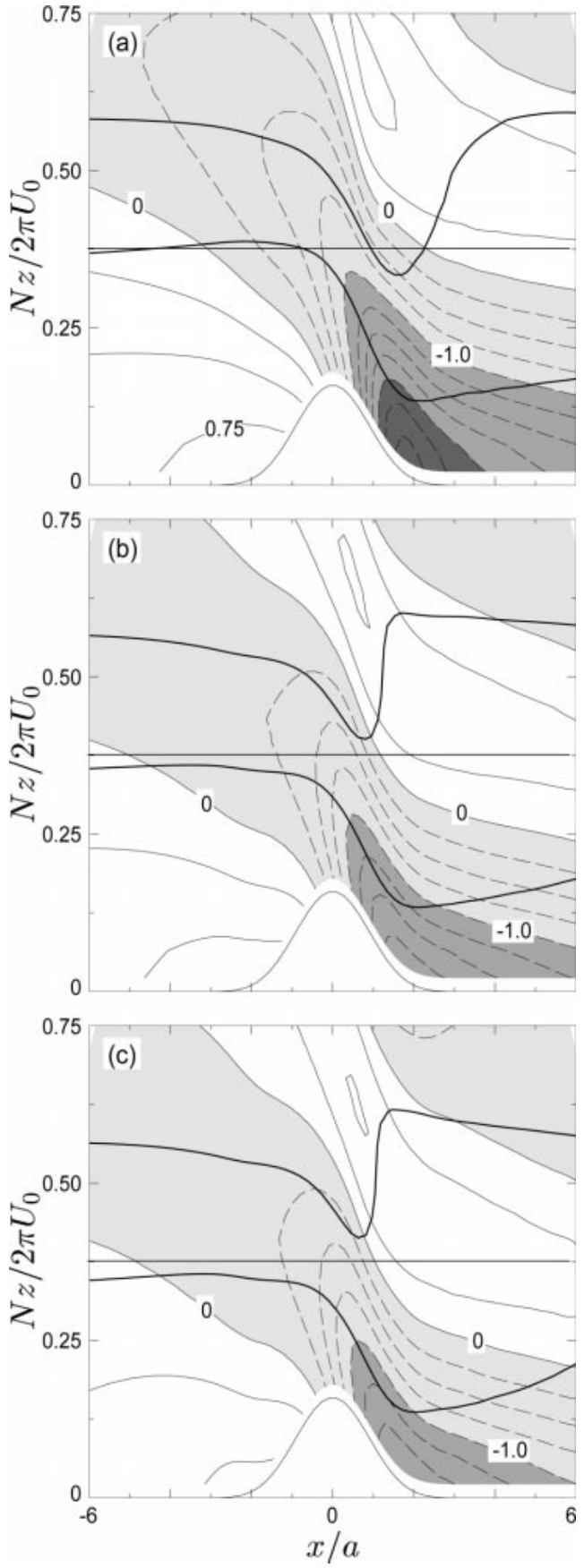

FIG. 10. As in Fig. 9 except that $P / \epsilon U_{0}^{2}$ is contoured at intervals of 0.25 . Darkest shading indicates values more negative than -2.0 .

case with $\epsilon=1.0$. The corresponding disturbance along the centerline of a 3D ridge with $\beta=7.5$ is shown in Fig. $11 \mathrm{~b}$. In the $2 \mathrm{D}$ case, the columnar modes propagate indefinitely upstream, with only a weak decay due to vertical dispersion. (As will be seen in Fig. 12, the lowlevel decelerated layer in Fig. 11a is still expanding upstream at $U_{0} t / a=40$.) In $3 \mathrm{D}$, the disturbance disperses in the horizontal as well as the vertical, and a
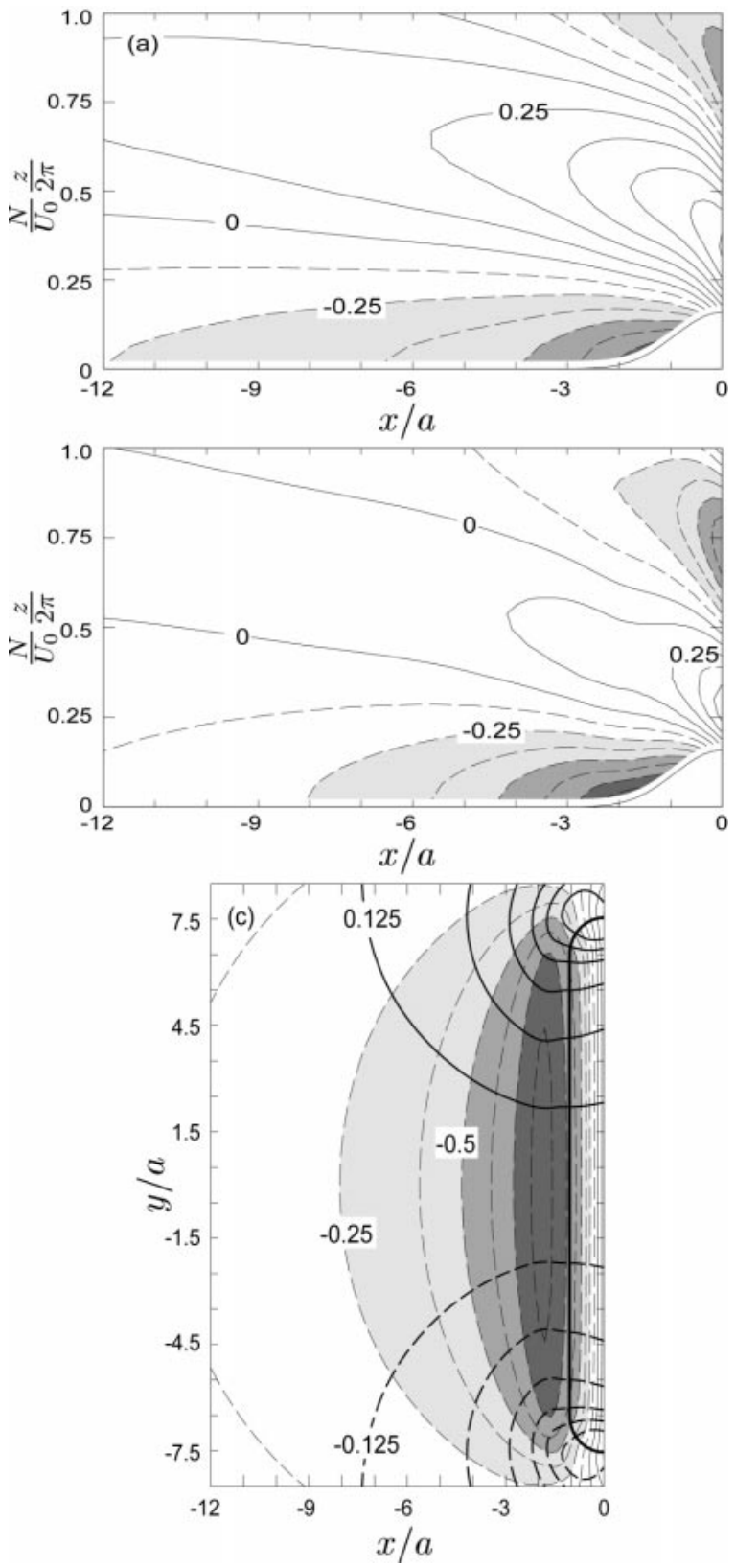

FIG. 11. The $x$ component disturbance wind speed $u / \epsilon U_{0}$ (contour interval 0.125 ; shading every 0.25 for negative values) upstream of the obstacle at $U_{0} t / a=40$ (a) for 2D ridge with $\epsilon=1.0$; (b) in cross section along the centerline $y=0$ of 3D ridge with $\beta=7.5$ and $\epsilon$ $=1.0$; and (c) at the lowest grid level for the $\beta=7.5$ and $\epsilon=1.0$ case. Heavy solid line in (c) is topographic contour at half maximum height. Also shown in (c) are contours of the $y$ component disturbance wind speed $v / \epsilon U_{0}$ (c.i., 0.125 ).

steady state is achieved with decelerated flow extending only a finite distance upstream. Figure $11 \mathrm{~b}$ shows that the magnitude of the disturbance wind speed (i.e., $|u|$ ) at the surface for $\beta=7.5$ decays upstream with a length scale slightly less than $\beta a$. This decay scale is consistent with the prediction of Baines (1995) (section 6.6) based 

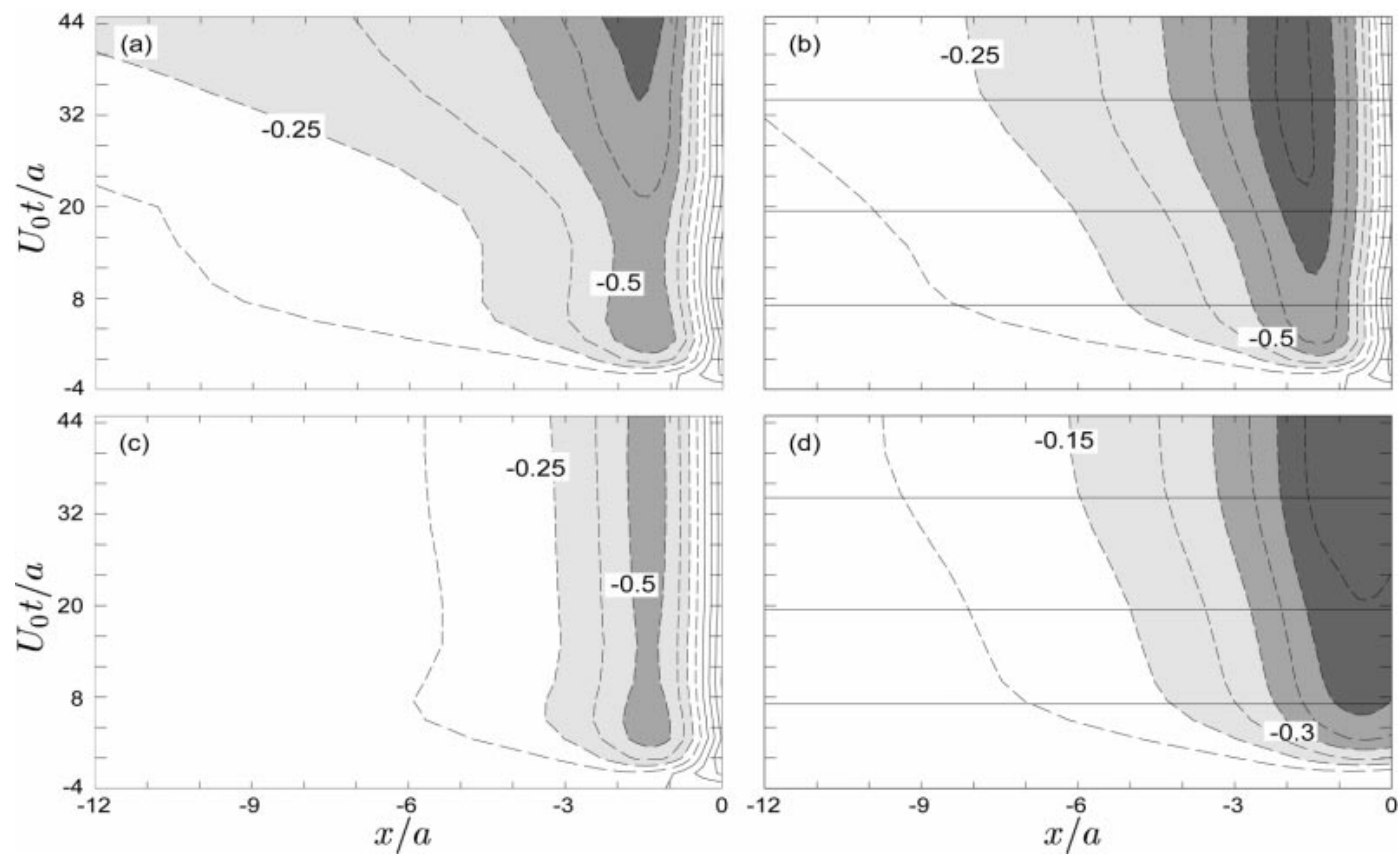

FIG. 12. The $x$ component disturbance wind speed $u / \epsilon U_{0}$ (c.i., 0.125 , shading every 0.25 for negative values) upstream of the obstacle at $z=h$ as a function of distance upstream and time (a) for 2D ridge with $\epsilon=1.0$; (b) at centerline $y$ $=0$ for 3D ridge with $\beta=7.5$ and $\epsilon=1.0$; (c) at $y=0$ for 3D ridge with $\beta=7.5$ and $\epsilon=0.5$; (d) shows $y$ component wind speed $v / \epsilon U_{0}$ (c.i., 0.075 , shading every 0.15$)$ at $z=h$ upstream of the end of the ridge at $y=-(\beta-1) a$ for the $\beta=7.5$ and $\epsilon=1.0$ case. Horizontal lines in (b) and (d) mark the local extrema in time of the surface pressure drag for $\beta=7.5$ and $\epsilon=1.0$ (cf. Fig. 13).

on small-amplitude theory for steadily forced columnar modes in 3D. Note that despite the limited upstream extent of the low-level decelerated layer in the 3D case, the minimum upstream $u$ is more negative for $\beta=7.5$ than for $2 \mathrm{D}$.

The horizontal structure of the disturbance at the lowest $u$ and $v$ grid level $\left(N z / U_{0} \approx \pi / 23\right)$ is shown in Fig. 11c. The contours of $v / \epsilon U_{0}$ show that the pattern of deflected flow at the ends of the ridge extends well upstream of the obstacle, suggesting that the enhanced low-level flow deflection in the high-drag state (cf. section $3 \mathrm{~b}$ ) is produced at least in part by the upstreampropagating modes. Figure 11c also shows that the flow deflection is accomplished without the occurrence of stagnant flow at the lowest $u$ and $v$ grid level (i.e., the minimum value in Fig. $11 \mathrm{c}$ is $u / U_{0}=-0.92>-1.0$ ).

The time development of the upstream disturbance in the $2 \mathrm{D}$ and $3 \mathrm{D}$ cases is considered in Fig. 12. Figures $12 \mathrm{a}$ and $12 \mathrm{~b}$ show the streamwise disturbance speed $u / \epsilon U_{0}$ at the surface along $y=0$ as a function of time for the $2 \mathrm{D}$ and $\beta=7.5$ cases with $\epsilon=1.0$. The case $\beta=7.5$ and $\epsilon=0.5$, in which wave breaking does not occur, is shown for comparison in Fig. 12c. All three cases exhibit a rapid upstream expansion of the region of decelerated flow associated with the transient growth of the disturbance over the time interval $-4 \leq U_{0} t / a$ $\leq 4$. The initial upstream surge is significantly stronger for $\epsilon=1.0$ than for $\epsilon=0.5$, but this difference cannot be attributed to wave breaking, since isentropes do not overturn in the $\epsilon=1.0$ cases until roughly $U_{0} t / a=7.5$. After the initial transient phase, the flow in the $\epsilon=0.5$ case becomes relatively steady, while in the $\epsilon=1.0$ cases, the flow continues to evolve in time.

In the $2 \mathrm{D}$ case with $\epsilon=1.0$, the most pronounced feature of the time development is the rapid advance of the upstream disturbance after $U_{0} t / a=20$ until the end of the simulation. Figs. $13 \mathrm{a}$ and $13 \mathrm{~b}$ show that the surface pressure drag is steadily amplifying during this same time interval. By contrast, during the earlier interval $8 \leq U_{0} t / a \leq 20$, the drag is decreasing in time and the upstream extent of the disturbance is nearly steady.

In the $\beta=7.5, \epsilon=1.0$ case, the surface drag after the initial transient phase is characterized by a damped oscillation about an apparent steady state. The frequency of the oscillation is a function of the width of the ridge with smaller $\beta$ producing a more rapid oscillation (not shown). As in the 2D case, the rate of expansion of the upstream disturbance appears to be related to the time tendency of the surface drag. The vertical lines in Figs. $13 \mathrm{a}$ and $13 \mathrm{~b}$ and the horizontal lines in Figs. 12b and $12 \mathrm{~d}$ mark the local maxima and minima in time of the surface drag for $\beta=7.5$. The times at which these maxima and minima occur divide the evolution of the upstream disturbance into distinct phases (Fig. 12b). During the interval $7 \leq U_{0} t / a \leq 20$, the drag is de- 

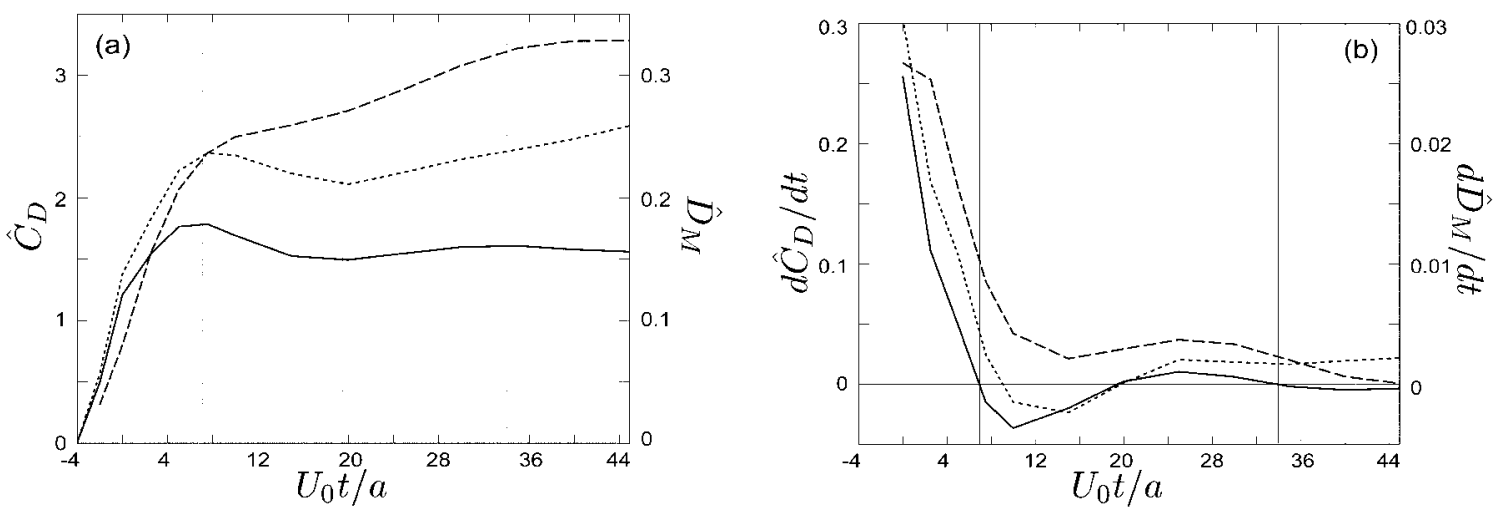

FIG. 13. (a) Nondimensional drag coefficient $\hat{C}_{D}$ as a function of time for the $2 \mathrm{D} \epsilon=1.0$ case (short dash) and for $\beta=7.5$ and $\epsilon=1.0$ (solid). Also shown is the scaled low-level fractional mass deflection $\hat{D}_{M}$ (long dash) for the $\beta=7.5$ case. (b) Time tendencies $\left(a / U_{0}\right) d \hat{C}_{D} / d t$ and $\left(a / U_{0}\right) d \hat{D}_{M} / d t$ for the curves shown in (a). Vertical lines in both panels mark the local extrema of the surface pressure drag for $\beta=7.5$ and $\epsilon=1.0$.

creasing in time, and the upstream advance of the lowlevel decelerated layer is slow. From $U_{0} t / a=20$ to $U_{0} t / a=34$, the drag is increasing, and the disturbance progresses upstream more rapidly. After $U_{0} t / a=34$, the drag shows a weak decrease (Fig. 13b), and the upstream advance of the decelerated layer is again slow.

Figure $12 \mathrm{~d}$ shows the cross-stream wind speed $v / \epsilon U_{0}$ at $z=h$ upstream of the end of the uniform height section of the ridge $[y=-(\beta-1) a]$ as a function of time for the $\beta=7.5$ and $\epsilon=1.0$ case. As the columnar disturbance propagates upstream, the region of deflected flow at the end of the ridge expands upstream as well. As a result, the low-level mass deflection $\hat{D}_{M}$ increases in time throughout the simulation. Note that the rate of increase in $\hat{D}_{M}$ (Fig. 13b) is well correlated with the rate at which the low-level decelerated layer expands upstream. This again suggests the importance of the upstream-propagating columnar disturbance in establishing the low-level mass deflection.

\section{Discussion: Limitations and extensions}

The results of the previous sections have been limited to the idealized case of nonrotating, free-slip flow with uniform basic wind and stability past smooth, isolated ridges. The consideration of such simplified prototype flows forms a basis for understanding more complex cases. Here we offer brief comments on the extension of our results to flows with nonuniform basic states and rotation.

In the case of uniform wind and stability, the transition to the high-drag state is concurrent with the steepening and overturning of isentropes above the lee slope. However, high-drag-state transitions are often produced without wave breaking in flows with height-varying basic stability. Preliminary computations using two uniform layers of different stability, as in Durran (1986a), suggest that the basic results of sections 3 and 4 extend to high-drag-state flows without wave breaking as well. The generation of upstream disturbances and the as- sociated low-level flow deflection are thus more intrinsically related to the high-drag-state transition than to wave breaking per se.

The extension of the present results to rotating flows past long ridges is perhaps more subtle. We restrict our remarks to the case of large Ro $=U_{0} / f a$ (e.g., Ro $\gtrsim$ 4) for which our results are most relevant. In the opposite limit of small Ro (i.e., Ro $\ll 1$ ) the flow is quasigeostrophic, and high-drag states do not occur. The intermediate regime of Ro $\sim 1$ has received relatively little attention in the literature (see, however, Trüb and Davies 1995), and we leave this case to future work.

For large Ro, the wave disturbance over the obstacle and presumably the mechanism for columnar mode generation are not directly affected by rotation. However, linear theory predicts that rotational effects limit the streamwise decay scale of an upstream columnar disturbance at steady state to roughly the deformation radius $L_{D}=N / m f$, where $m$ is the dominant vertical wavenumber of the upstream-propagating modes. [Note that there is in general no a priori way to predict an appropriate value for $m$. Pierrehumbert and Wyman (1985) suggest $m \approx \gamma / h_{0}$, where $\gamma$ is some universal constant; alternatively, from Fig. 11a, we might estimate $m \approx$ $2 N / 3 U_{0}$ ).] As discussed in section 4, horizontal dispersion limits the upstream decay scale to roughly $\beta a$. We thus expect that for $\beta a<L_{D}$, rotation has little effect on either the mountain wave in the vicinity of the obstacle or the upstream disturbance, and the results of the present study apply without modification. By contrast, for $\beta a>L_{D}$ the upstream decay scale is limited by rotation, and the upstream disturbance adjusts toward geostrophy with a greater fraction of the flow deflected northward around the ridge. The modification of the upstream flow then likely affects the wave disturbance over the obstacle as well. Nonetheless, it seems probable that the basic result of increased flow deflection and consequent increase in $3 \mathrm{D}$ effects with transition to the high-drag state still applies. 


\section{Summary and conclusions}

This study has compared flow over ridges in $3 \mathrm{D}$ to the well-studied 2D limit. Our results indicate that for linear and weakly nonlinear cases, the low-level flow over the interior of the ridge is well described by $2 \mathrm{D}$ theory when $\beta$ is roughly 10 or greater. By constrast, when $\epsilon$ is large enough to force wave breaking and highdrag-state flow, substantial discrepancies between the $2 \mathrm{D}$ and $3 \mathrm{D}$ results remain apparent for the largest $\beta$ considered in this work $(\beta=12)$.

As in the 2D case, an abrupt increase in the surface form drag occurs when the height of an elongated ( $\beta$ $>1$ ) 3D ridge is made large enough to force overturning isentropes. The results of this study show that in 3D, the transition to the high-drag state is also accompanied by an abrupt increase in low-level flow deflection. Even at $\beta=12$, a significant fraction of the incident lowlevel mass flux in the high-drag state is deflected around the ridge rather than ascending the crest. The increased flow deflection is produced at least in part by the upstream-propagating columnar disturbances associated with the high-drag-state transition. As a result of the low-level flow deflection, the enhancement of the surface drag associated with wave breaking is significantly weaker for the $3 \mathrm{D}$ case than for $2 \mathrm{D}$. For example, the nonlinear enhancement of the surface drag as $\epsilon$ is increased above the critical value for wave breaking in $2 \mathrm{D}$ is roughly twice the corresponding enhancement for $\beta=7.5$.

The pronounced low-level flow deflection in the highdrag state leads to important morphological differences between the 2D and 3D flows. The 3D case features warmer near-surface flow above the lee slope, as air from aloft descends to replace the deflected mass. At the same time, the vertical displacement of fluid particles over the windward slope is weaker in the 3D case, since less of the incident mass ascends to the crest. The reduced vertical displacement above the windward slope leads to weaker wave amplitude and weaker buoyancy perturbations aloft. As a result, the vertically integrated buoyancy above the lee slope and the magnitude of the lee-side surface pressure minimum are less for 3D highdrag-state flow than for 2D. The weakening of the leeside surface pressure minimum primarily accounts for the weaker cross-mountain pressure drag in the 3D case.

In the present work, we have carefully diagnosed and explored the onset of 3D effects with increasing $\epsilon$ in flows over long ridges. Most previous studies have emphasized the importance of windward-slope stagnation in establishing 3D flow perturbations. In particular, it has been suggested that stagnation on the upstream slope produces a transition from a regime in which flow passes directly over the obstacle to a more fully $3 \mathrm{D}$ regime in which flow is deflected laterally at low levels (e.g., Smith 1989a; Baines 1995, section 6.7). Smith and Grønås (1993) and Baines (1995, sections 6.4, 6.7) have further suggested that wave breaking promotes stag- nation on the windward slope (at least for relatively small $\beta$ ) and thereby induces the transition to the flowsplitting regime. For long ridges (large $\beta$ ), the present results show that wave breaking may introduce significant low-level flow deflection and consequent 3D effects without windward stagnation. In the $\beta=7.5$ case shown in Figs. 3 and 7, upstream stagnation first occurs at the surface for $\epsilon=1.0 .^{6}$ In contrast to the evident effects of wave breaking at $\epsilon=0.8$, the occurrence of upstream stagnation has no noticeable effect on the surface drag or the low-level mass deflection.

Acknowledgments. This research was supported by National Science Foundation grants ATM-9530662 and ATM-9817728. Part of this work was completed while CCE was the recipient of a National Defense Science and Engineering Graduate Fellowship.

\section{APPENDIX}

\section{Time Variation of the Surface Drag after the Transient Growth Phase}

Figures A1a-c indicate the time variation of the surface drag [in terms of the nondimensional drag coefficient $\hat{C}_{D}$ defined in (9)] after the transient growth phase for a $3 \mathrm{D}$ ridge with $\beta=7.5$ and 3 different values of $\epsilon$. Thin horizontal lines give the chosen representative quasi-steady value for each case. In the $\epsilon=0.5$ case of Fig. A1a, the obstacle height is below the critical value at which isentropes overturn and wave breaking occurs. By contrast, wave breaking is present in both the $\epsilon=0.8$ and $\epsilon=1.0$ cases of Figs. A1b and A1c. Figure A1d shows an example of a $2 \mathrm{D}$ case with wave breaking $(\epsilon=1.0)$.

In the $3 \mathrm{D}$ cases without breaking waves, the time variation of the surface drag is minimal after roughly $U_{0} t / a=15$, and the steady-state values are thus determined rather precisely. In the example of Fig. A1a, the range in the drag after $U_{0} t / a=15$ is less than $\pm 1 \%$ of the chosen representative value. Similar precision is obtained in the 2D cases without wave breaking. However, when wave breaking occurs, the surface drag is less steady. For 3D ridges, the greatest time variation in the surface drag after the transient growth phase is observed for long ridges with heights just greater than the threshold at which breaking waves are forced. The $\beta=7.5$

\footnotetext{
${ }^{6}$ Note that stagnant flow at the lowest grid level for $u$ and $v\left(N z / U_{0}\right.$ $\approx \pi / 23$ ) does not occur at steady state until $\epsilon=1.3$ (cf. Fig. 11c). However, a more precise diagnosis of surface stagnation may be obtained from consideration of $w$, which is defined at the lower boundary. Along the centerline of the obstacle, $v=0$, so that by (4), $w=$ $\left(u_{0}+u\right) \partial h / \partial x$ at $z=h$. The occurrence of $w \leq 0$ along the centerline on the upstream slope then implies $u_{0}+u \leq 0$ as well. Using this more precise criterion, surface stagnation first occurs at $\epsilon=1.0$ (but does not occur for the $\epsilon=0.8$ or $\epsilon=0.9$ cases, both of which feature breaking waves and significant upstream deflection).
} 

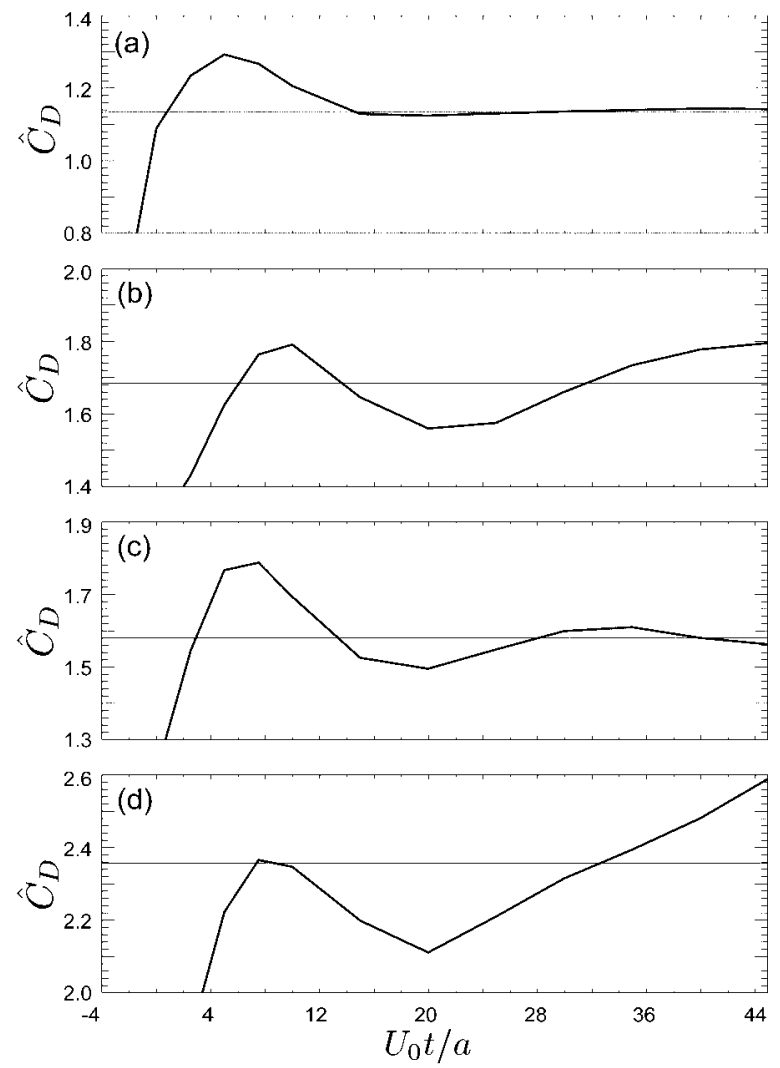

FIG. A1. Time variation of the surface drag after the initial transient phase. Heavy lines show the nondimensional drag coefficient $\hat{C}_{D}$ [defined in (9)], and thin horizontal lines show the value taken as the representative quasi-steady value. (a)-(c) show results for $\beta=7.5$ and (a) $\epsilon=0.5$, (b) $\epsilon=0.8$, and (c) $\epsilon=1.0$; (d) shows the 2D case for $\epsilon=1.0$

and $\epsilon=0.8$ case in Fig. A1b is an example of such a flow. Note that even in this case, the range in the drag after the initial growth phase is limited to roughly $\pm 7 \%$ of the representative quasi-steady value. As the height of a 3D ridge is further increased, the drag becomes somewhat more steady. In the $\beta=7.5$ and $\epsilon=1.0$ case of Fig. A1c, the range in the drag after $U_{0} t / a=20$ is at most $\pm 5 \%$; furthermore, the oscillation in the drag decays in time so that the steady-state value is likely determined to within a few percent. By contrast, when wave breaking occurs in the $2 \mathrm{D}$ cases, the drag steadily amplifies after $U_{0} t / a=20$ and does not approach a quasi-steady state (Fig. A1d). In this case, we take the drag at $U_{0} t / a=32.5$ as a representative value.

\section{REFERENCES}

Afanasyev, Y. D., and W. R. Peltier, 1998: The three-dimensionalization of stratified flow over two-dimensional topography. $J$. Atmos. Sci., 55, 19-39.

Andreassen, Ø., C. E. Wasberg, D. C. Fritts, and J. R. Isler, 1994: Gravity wave breaking in two and three dimensions. 1: Model description and comparison of two-dimensional evolutions. $J$. Geophys. Res., 99, 8095-8108.
Bacmeister, J. T., and R. T. Pierrehumbert, 1988: On high-drag states of nonlinear stratified flow over an obstacle. J. Atmos. Sci., 45, 63-80.

Baines, P. G., 1995: Topographic Effects in Stratified Flows. Cambridge University Press, $482 \mathrm{pp}$.

Batchelor, G. K., 1967: An Introduction to Fluid Dynamics. Cambridge University Press, 615 pp.

Bauer, M. H., G. J. Mayr, I. Vergeiner, and H. Pichler, 2000: Strongly nonlinear flow over and around a three-dimensional mountain as a function of the horizontal aspect ratio. J. Atmos. Sci., 57, 3971-3991.

Bougeault, P., 1983: A non-reflective upper boundary condition for limited-height hydrostatic models. Mon. Wea. Rev., 111, 420429.

Clark, T. L., and W. R. Peltier, 1977: On the evolution and stability of finite-amplitude mountain waves. J. Atmos. Sci., 34, 17151730.

Durran, D. R., 1986a: Another look at downslope windstorms. Part I: The development of analogs to supercritical flow in an infinitely deep, continously stratified fluid. J. Atmos. Sci., 43, 25272543.

_- 1986b: Mountain waves. Mesoscale Meteorology and Forecasting, P. S. Ray, Ed., Amer. Meteor. Soc., 472-492.

_ 1995: Do breaking mountain waves decelerate the local mean flow? J. Atmos. Sci., 52, 4010-4032.

— of moist mountain waves. Mon. Wea. Rev., 111, 2341-2361.

— Nonlinear amplification beneath wave-overturning layers. $J$. Atmos. Sci., 44, 3402-3412.

Epifanio, C. C., 1999: High-drag states and lee vortices in stratified flow over topography. Ph.D. dissertation, University of Washington, $97 \mathrm{pp}$.

Gal-Chen, T., and R. Somerville, 1975: On the use of a coordinate transformation for the solution of the Navier-Stokes equations. J. Comput. Phys., 17, 209-228.

Klemp, J. B., and D. R. Durran, 1983: An upper boundary condition permitting internal gravity wave radiation in numerical mesoscale models. Mon. Wea. Rev., 111, 430-444.

Lilly, D. K., 1962: On the numerical simulation of buoyant convection. Tellus, 14, 148-172.

Miranda, P. M. A., and I. N. James, 1992: Non-linear three-dimensional effects on gravity-wave drag: Splitting flow and breaking waves. Quart. J. Roy. Meteor. Soc., 118, 1057-1081.

Ólafsson, H., and P. Bougeault, 1996: Nonlinear flow past an elliptic mountain ridge. J. Atmos. Sci., 53, 2465-2489.

— , and - 1997: The effect of rotation and surface friction on orographic drag. J. Atmos. Sci., 54, 193-210.

Pearson, R., 1974: Consistent boundary conditions for numerical models of systems that admit dispersive waves. J. Atmos. Sci., 31, 1481-1489.

Peltier, W. R., and T. L. Clark, 1979: The evolution and stability of finite-amplitude mountain waves. Part II: Surface wave drag and severe downslope windstorms. J. Atmos. Sci., 36, 1498-1529.

Pierrehumbert, R. T., and B. Wyman, 1985: Upstream effects of mesoscale mountains. J. Atmos. Sci., 42, 977-1003.

Schär, C., and D. R. Durran, 1997: Vortex formation and vortex shedding in continously stratified flows past isolated topography. J. Atmos. Sci., 54, 534-554.

Skamarock, W. C., and J. Klemp, 1993: Adaptive grid refinement for two-dimensional and three-dimensional nonhydrostatic atmospheric flow. Mon. Wea. Rev., 121, 788-804.

Smith, R. B., 1979: The influence of mountains on the atmosphere. Advances in Geophysics, Vol. 21, Academic Press, 87-230.

, 1980: Linear theory of stratified hydrostatic flow past an isolated mountain. Tellus, 32, 348-364.

- 1985: On severe downslope winds. J. Atmos. Sci., 42, 25972603.

- 1988: Linear theory of stratified flow past an isolated mountain in isosteric coordinates. J. Atmos. Sci., 45, 3889-3896. 
_ 1989a: Hydrostatic airflow over mountains. Advances in Geophysics, Vol. 31, Academic Press, 1-41.

$\ldots, 1989$ b: Mountain induced stagnation points in hydrostatic flow. Tellus, 41A, 270-274.

_ _ and S. Grønås, 1993: Stagnation points and bifurcation in 3-D mountain airflow. Tellus, 45A, 28-43.

Smolarkiewicz, P. K., and R. Rotunno, 1989: Low Froude number flow past three-dimensional obstacles. Part I: Baroclinically generated lee vortices. J. Atmos. Sci., 46, 1154-1164.

_ , and -1990 : Low Froude number flow past three-dimensional obstacles. Part II: Upwind flow reversal zone. J. Atmos. Sci., 47, 1498-1511.

Trüb, J., and H. C. Davies, 1995: Flow over a mesoscale ridge: Pathways to regime transition. Tellus, $\mathbf{4 7 A}, 502-524$. 